\title{
Distributed user selection scheme for uplink multiuser MIMO systems in a multicell environment
}

\author{
Byong Ok Lee ${ }^{1}$, Oh-Soon Shin ${ }^{2^{*}}$ and Kwang Bok Lee ${ }^{3}$
}

\begin{abstract}
We propose an interference-aware user selection scheme for uplink multiuser multiple-input multiple-output systems in a multicell environment. The proposed scheme works in a distributed manner. Each mobile station determines its transmit beamforming vector based on the locally available channel state information, and informs the associated base station (BS) of the amount of potential interference caused to adjacent cells along with the resulting beamforming vector. Then, the BS selects a set of users to be served simultaneously with consideration of intercell interference. The user selection scheme is devised either to maximize the sum rate or to achieve proportional fairness among users. For each case, we derive an optimal user selection criterion and propose a suboptimal distributed user selection algorithm with low complexity. Simulation results confirm that the proposed scheme offers significant throughput enhancement due to reduction of the intercell interference in a multicell environment.
\end{abstract}

\section{Introduction}

Multiuser multiple-input multiple-output (MU-MIMO) is widely accepted as a key technology for enabling highspeed wireless access. In the uplink MU-MIMO systems, multiple mobile stations (MSs) are allowed to simultaneously transmit their signals to the base station (BS) to increase the system capacity. Under this scenario, the system performance may depend on the set of transmitting users and their transmit beamforming vectors [1-3]. In [1], a general framework for transmit beamforming and user selection was developed based upon general convex utility functions. In [2], successive user selection algorithms were proposed along with optimization of transmit beamforming vectors. In [3], various lowcomplexity beamforming and user selection schemes were proposed. All these works, however, have dealt with only a single cell environment where the intercell interference does not exist.

Intercell interference is one of the most critical factors that limit the performance of cellular systems, especially for low-frequency reuse factor. There have been several

\footnotetext{
* Correspondence: osshin@ssu.ac.kr

${ }^{2}$ School of Electronic Engineering, Soongsil University, Seoul 156-743, South Korea

Full list of author information is available at the end of the article
}

works on MIMO that account for the intercell interference in a multicell environment [4-7]. In [4], it was reported that the performance of spatial multiplexing MIMO scheme is significantly degraded in an interference-limited multicell environment. In [5], an optimal MIMO transmission strategy was studied when the channel state information (CSI) is not available at the transmitter. For the case when the CSI is available at the transmitter, a centralized precoding scheme that maximizes the total sum rate was proposed in [6]. In [7], a precoding scheme was proposed to maximize the total sum rate in a distributed manner. However, these works have been based on a single user MIMO system where only one MS is served at a time. MU-MIMO systems were only recently investigated in a multicell environment [8-10]. In [8], downlink multicell MU-MIMO systems were discussed from the aspects of tradeoffs, overhead, and interference control. In [9], scheduling schemes were developed for the downlink multicell MIMO systems. Uplink MU-MIMO systems were analyzed in [10] in the case that the adjacent BS's are allowed to cooperate.

In this article, we develop an interference-aware user selection scheme for uplink MU-MIMO systems in a 
multicell environment. The scheme comprises of two steps and works in a distributed manner. In the first step, each MS determines its transmit beamforming vector. By utilizing the previous result on the interferenceaware beamforming proposed in [7], we can effectively reduce the interference caused to adjacent cells. In the second step, each BS selects a set of users to be served simultaneously to realize multiuser diversity with consideration of interference caused to adjacent cells as well as the desired link performance. The user selection scheme is developed so to maximize the sum rate or to achieve proportional fairness among users. For each objective, we derive an optimal user selection criterion and propose a suboptimal distributed user selection algorithm with low complexity. Simulation results are provided to show the throughput enhancement of the proposed scheme.

The rest of this article is organized as follows. Section 2 describes the system model. In Section 3, we explain distributed beamforming schemes. In Section 4, we propose an uplink user selection algorithm based on the beamforming vectors. Simulation results are presented in Section 5, and conclusions are drawn in Section 6.

We define here some notation used throughout this article. We use boldface capital letters and boldface small letters to denote matrices and vectors, respectively, $(\cdot)^{\mathrm{T}}$ and $(\cdot)^{\mathrm{H}}$ to denote transpose and conjugate transpose, respectively, $\operatorname{det}(\cdot)$ to denote determinant of a matrix, $\operatorname{tr}(\cdot)$ to denote trace of a matrix, $(\cdot)^{-1}$ to denote matrix inversion, $\|\cdot\|$ to denote Euclidean norm of a vector, $\mathbf{I}_{N}$ to denote the $N \times N$ identity matrix.

\section{System model}

We consider the uplink of an MU-MIMO system comprised of $L$ cells where there are $K$ users in each cell. Each MS and each BS are equipped with $N_{t}$ transmit antennas and $N_{r}$ receive antennas, respectively. The $k$ th MS in the $i$ th cell is assumed to communicate with the BS in the $i$ th cell by using a transmit beamforming vector $\mathbf{w}_{i}^{(k)}$.

The received signal vector $\mathbf{y}_{i}$ at the BS in the $i$ th cell can be expressed as

$$
\begin{aligned}
\mathbf{y}_{i} & =\sum_{k \in \mathbf{S}_{i}} \sqrt{\rho_{i}^{(k)}} \mathbf{H}_{i, i}^{(k)} \mathbf{w}_{i}^{(k)} x_{i}^{(k)} \\
& +\sum_{j=1, j \neq i}^{L} \sum_{k \in \mathbf{S}_{j}} \sqrt{\eta_{i, j}^{(k)}} \mathbf{H}_{i, j}^{(k)} \mathbf{w}_{j}^{(k)} x_{j}^{(k)}+\mathbf{n}_{i},
\end{aligned}
$$

where $\mathbf{S}_{i}$ denotes the set of selected users to be simultaneously served in the $i$ th cell. We assume that the maximum number of selected users per cell is $N_{r} . x_{i}^{(k)}$ denotes the input symbol transmitted from the $k$ th MS in the $i$ th cell, $\mathbf{H}_{i, j}^{(k)}$ denotes an $N_{r} \times N_{t}$ channel matrix between the $k$ th MS in the $j$ th cell and the BS in the $i$ th cell. We assume a flat fading channel in both time and frequency. The elements of $\mathbf{H}_{i, j}^{(k)}$ and $x_{i}^{(k)}$ are assumed to be independent and identically distributed (i.i.d.) circularly symmetric complex Gaussian random variables with zero mean and unit variance. In (1), $\mathbf{n}_{i}$ denotes the additive white Gaussian noise (AWGN) vector at the BS in the $i$ th cell with each element having unit variance, $\rho_{i}^{(k)}$ denotes the signal-to-noise ratio (SNR) of the $k$ th MS in the $i$ th cell, and $\eta_{i, j}^{(k)}$ denotes the interference-tonoise ratio (INR) for the interference that the $k$ th MS in the $j$ th cell causes to the BS in the $i$ th cell.

We assume that each BS performs a linear minimum mean-square error (MMSE) detection to suppress the residual interference and detect the desired signal. The MMSE combining vector $\mathbf{g}_{i}^{(k)}$ used in receiving the $k$ th MS's signal in the $i$ th cell is expressed as

$$
\mathbf{g}_{i}^{(k)}=\left(\sqrt{\rho_{i}^{(k)}} \mathbf{H}_{i, i}^{(k)} \mathbf{w}_{i}^{(k)}\right)^{H}\left(\mathbf{K}_{\mathrm{NI}}^{(i, k)}\right)^{-1}
$$

where $\mathbf{K}_{\mathrm{NI}}^{(i, k)}$ denotes the covariance matrix of the noise plus received interference signal which is given as

$$
\begin{aligned}
\mathbf{K}_{\mathrm{NI}}^{(i, k)}= & \mathbf{I}_{N_{r}} \\
& +\sum_{k^{\prime} \in \mathbf{S}_{i}, k^{\prime} \neq k} \rho_{i}^{\left(k^{\prime}\right)}\left(\mathbf{H}_{i, i}^{\left(k^{\prime}\right)} \mathbf{w}_{i}^{\left(k^{\prime}\right)}\right)\left(\mathbf{H}_{i, i}^{\left(k^{\prime}\right)} \mathbf{w}_{i}^{\left(k^{\prime}\right)}\right)^{H} \\
& +\sum_{j=1, j \neq i}^{L} \sum_{k^{\prime} \in \mathbf{S}_{j}} \eta_{i, j}^{\left(k^{\prime}\right)}\left(\mathbf{H}_{i, j}^{\left(k^{\prime}\right)} \mathbf{w}_{j}^{\left(k^{\prime}\right)}\right)\left(\mathbf{H}_{i, j}^{\left(k^{\prime}\right)} \mathbf{w}_{j}^{\left(k^{\prime}\right)}\right)^{H} .
\end{aligned}
$$

In (3), the first term is due to the AWGN, and the second and third terms represent the intracell interference and intercell interference, respectively. The post processing SINR of the $k$ th MS's signal in the $i$ th cell is represented as

$$
\operatorname{SINR}_{i}^{(k)}=\left(\sqrt{\rho_{i}^{(k)}} \mathbf{H}_{i, i}^{(k)} \mathbf{w}_{i}^{(k)}\right)^{H}\left(\mathbf{K}_{\mathrm{NI}}^{(i, k)}\right)^{-1}\left(\sqrt{\rho_{i}^{(k)}} \mathbf{H}_{i, i}^{(k)} \mathbf{w}_{i}^{(k)}\right) .
$$

Then, the achievable rate of the $k$ th MS in the $i$ th cell is calculated as

$$
r_{i}^{(k)}=\log \left(1+\operatorname{SINR}_{i}^{(k)}\right) .
$$

Since the achievable rate is affected by the intercell interference, the optimal design for transmit beamforming and user selection needs a system-wide centralized optimization, which requires a lot of feedback and huge signaling overhead among cells, making the algorithm impractical. Instead of a centralized approach, we take a 
distributed approach for determining transmit beamforming vectors and the corresponding set of users, as illustrated in Figure 1. In the first step, each MS determines its transmit beamforming vector based on the locally available CSI and calculates the amount of potential interference caused to adjacent cells. Then, each MS informs the associated BS of the amount of interference to adjacent cells along with the determined beamforming vector. In the second step, each BS selects a set of users to be simultaneously served based on the information received from MSs. The BS then broadcasts the indices of selected users with appropriate modulation and coding schemes level. Finally, the selected users transmit their own data to the BS. It must be noted that the proposed approach based on the local CSI will provide a more practical solution than the centralized optimization from the viewpoint of feedback overhead and computational complexity, although it may not guarantee the optimality. We explain details of the transmit beamforming and user selection scheme in the following two sections.

\section{Transmit beamforming}

In this section, we explain transmit beamforming schemes that were proposed in [7] for the case of single user MIMO in a multicell environment. We assume that each MS independently determines its transmit beamforming vector based on the locally available CSI.
We define the desired channel $\mathbf{H}_{\mathrm{D}}^{(i, k)}$ and interference generating channel $\mathbf{H}_{\mathrm{GI}}^{(i, k)}$ for the $k$ th $\mathrm{MS}$ in the $i$ th cell as

$$
\begin{aligned}
\mathbf{H}_{\mathrm{D}}^{(i, k)}= & \sqrt{\rho_{i}^{(k)}} \mathbf{H}_{i, i}^{(k)}, \\
\mathbf{H}_{\mathrm{GI}}^{(i, k)}= & {\left[\begin{array}{c}
\sqrt{\eta_{1, i}^{(k)}} \mathbf{H}_{1, i}^{(k)} \\
\vdots \\
\sqrt{\eta_{i-1, i}^{(k)}} \mathbf{H}_{i-1, i}^{(k)} \\
\sqrt{\eta_{i+1, i}^{(k)}} \mathbf{H}_{i+1, i}^{(k)} \\
\vdots \\
\sqrt{\eta_{L, i}^{(k)}} \mathbf{H}_{L, i}^{(k)}
\end{array}\right] . }
\end{aligned}
$$

We assume that the $k$ th MS can obtain $\mathbf{H}_{\mathrm{D}}^{(i, k)}$ and $\mathbf{H}_{\mathrm{GI}}^{(i, k)}{ }^{(i, k)} \mathbf{H}_{\mathrm{GI}}$ by exploiting the channel reciprocity. This is possible for time division duplex systems. For example, the MS in the $i$ th cell can estimate $\mathbf{H}_{\mathrm{D}}^{(i, k)}$ through downlink signal that comes from the BS in the $i$ th cell. Similarly, the MS can determine $\mathbf{H}_{\mathrm{GI}}^{(i, k)}{ }^{(i, k)} \mathbf{H}_{\mathrm{GI}}$ by estimating the covariance matrix of aggregate interference signals that come from adjacent cells during the downlink period. Based on the above assumptions, we introduce two distributed transmit beamforming schemes proposed in [7]: MAX-SNR beamforming and MAX-SGINR beamforming.

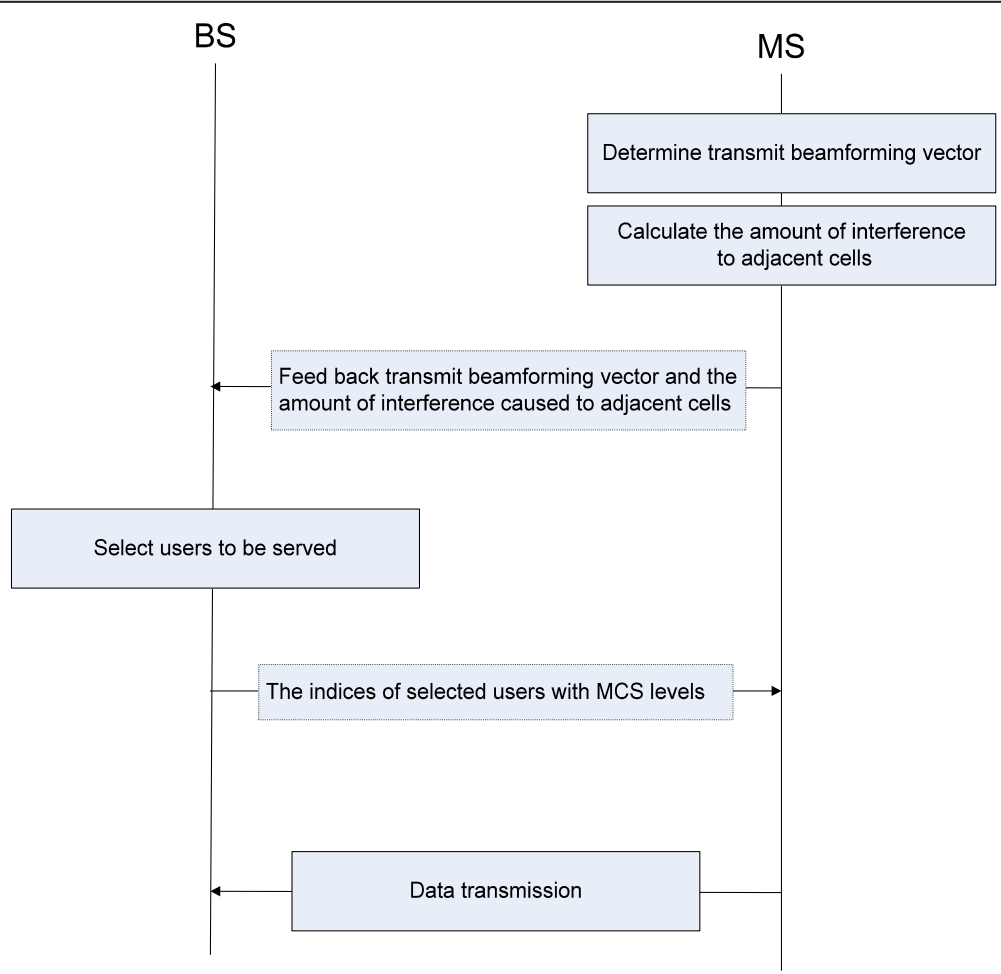

Figure 1 The proposed approach for distributed transmit beamforming and user selection. 


\section{MAX-SNR beamforming}

The MAX-SNR beamforming vector is constructed to maximize the desired signal power without consideration on the intercell interference. The MAX-SNR beamforming vector of the $k$ th MS in the $i$ th cell can be expressed as

$$
\mathbf{w}_{\mathrm{SNR} i}^{(k)}=\underset{\mathbf{w}_{i}^{(k)}}{\arg \max }\left\|\mathbf{H}_{\mathrm{D}}^{(i, k)} \mathbf{w}_{i}^{(k)}\right\|^{2} \text { s.t. }\left\|\mathbf{w}_{i}^{(k)}\right\|^{2}=1 .
$$

The solution of (8) can be obtained as the eigenvector corresponding to the largest eigenvalue of $\mathbf{H}_{\mathrm{D}}^{(i, k) H} \mathbf{H}_{\mathrm{D}}^{(i, k)}$.

\section{MAX-SGINR beamforming}

The MAX-SGINR beamforming vector is determined considering not only the desired signal power, but also the intercell interference caused to adjacent cells. The metric called signal to generated interference plus noise ratio (SGINR) at the $k$ th MS in the $i$ th cell is defined as

$$
\operatorname{SGINR}_{i}^{(k)}=\frac{\left\|\mathbf{H}_{\mathrm{D}}^{(i, k)} \mathbf{w}_{i}^{(k)}\right\|^{2}}{1+\left\|\mathbf{H}_{\mathrm{GI}}^{(i, k)} \mathbf{w}_{i}^{(k)}\right\|^{2}},
$$

where the numerator corresponds to the desired signal power and the denominator represents the noise plus interference caused to adjacent cells by the kth MS in the $i$ th cell. The MAX-SGINR beamforming vector maximizes the SGINR at each MS as

$$
\begin{aligned}
\mathbf{w}_{\mathrm{SGINR} i}^{(k)} & =\frac{\arg \max _{\mathbf{w}_{i}^{(k)}}\left\|\mathbf{H}_{\mathrm{D}}^{(i, k)} \mathbf{w}_{i}^{(k)}\right\|^{2}}{1+\left\|\mathbf{H}_{\mathrm{GI}}^{(i, k)} \mathbf{w}_{i}^{(k)}\right\|^{2}} \text { s.t. }\left\|\mathbf{w}_{i}^{(k)}\right\|^{2} \\
& =1 .
\end{aligned}
$$

The solution of (10) can be obtained as the eigenvector corresponding to the largest eigenvalue of $\left(\mathbf{I}_{N_{t}}+\mathbf{H}_{\mathrm{GI}}^{(i, k)}{ }^{H} \mathbf{H}_{\mathrm{GI}}^{(i, k)}\right)^{-1} \mathbf{H}_{\mathrm{D}}^{(i, k)}{ }^{H} \mathbf{H}_{\mathrm{D}}^{(i, k)}$. The MAX-SGINR beamforming effectively reduces the interference to adjacent cells while maintaining the desired signal power. It is shown in [7] that the MAX-SGINR beamforming approximately maximizes the total sum rate for multipleinput single-output systems in a two-cell environment.

After determining a transmit beamforming vector, each MS calculates the amount of interference caused to adjacent cells as

$$
\beta_{i}^{(k)}=\left\|\mathbf{H}_{\mathrm{GI}}^{(i, k)} \mathbf{w}_{i}^{(k)}\right\|^{2}
$$

where $\beta_{i}^{(k)}$ denotes the amount of interference caused to adjacent cell by the $k$ th $\mathrm{MS}$ in the $i$ th cell. Note that $\beta_{i}^{(k)}$ depends on the transmit beamforming vector. Each MS informs the associated BS of $\mathbf{w}_{i}^{(k)}$ and $\beta_{i}^{(k)}$ for user selection.

\section{User selection}

In this section, we develop user selection schemes with two different objectives: sum rate maximization, and proportional fairness (PF). For each objective, we first derive an optimal user selection criterion and then propose a suboptimal distributed algorithm with low complexity.

\section{Sum rate maximization}

We begin with a conventional user selection algorithm for sum rate maximization, which was proposed for a single cell environment. In this case, each BS selects users to maximize only the sum rate of its own cell as

$$
\mathbf{S}_{\mathrm{CONV} i}=\underset{\mathbf{S}_{i}}{\arg \max } \sum_{k \in \mathbf{S}_{i}} r_{i}^{(k)} \text { for } i=1,2, \ldots, L .
$$

However, this solution is not optimal in a multicell environment due to the intercell interference. In order to maximize the total sum rate of the $L$ cells, we modify the formulation of (12) as

$$
\left(\mathbf{S}_{\mathrm{opt} 1}, \mathbf{S}_{\mathrm{opt} 2}, \ldots, \mathbf{S}_{\mathrm{opt} L}\right)=\underset{\left(\mathbf{S}_{1}, \mathbf{S}_{2}, . . \mathbf{S}_{L}\right)}{\arg \max } \sum_{i=1}^{L} \sum_{k \in \mathbf{S}_{i}} r_{i}^{(k)} .
$$

The solution of (13) can only be obtained through centralized optimization among cells, which requires perfect CSI, a lot of signaling overhead among cells, and very high computational complexity. As a more practical solution, we propose a suboptimal distributed user selection algorithm with low complexity. The algorithm is described as in the following steps.

Step 1. Initialization: $\mathbf{S}_{i}=\{\}$.

Step 2. $k_{\text {new }}=\arg \max _{k} \Delta C(k)$.

Step 3. If $\Delta C\left(k_{\text {new }}\right)>0$, then $\mathbf{S}_{i}=\mathbf{S}_{i} \cup\left\{k_{\text {new }}\right\}$ and go

back to the Step 2; otherwise terminate the algorithm.

Each BS independently selects users to be served by using the above algorithm. In Step 1, the set $S_{i}$ of selected users is initialized. In Step 2, the BS chooses one user among the users not in $S_{i}$ so as to maximize the amount of the change in the total sum rate. Note that $\Delta C(k)$ denotes the amount of the change in the total sum rate when the $k$ th user is added to $S_{i}$. In Step 3 , if the addition of the selected user in Step 2 increases the total sum rate, then the BS adds the user to $S_{i}$ and goes back to Step 2. Otherwise, the algorithm terminates and the final set of selected users is given by $S_{i}$.

The most challenging part of the above algorithm is to calculate $\Delta C(k)$ without sharing information among 
neighboring cells. We can split $\Delta C(k)$ into two components as

$$
\Delta C(k)=\Delta C_{\text {gain }}(k)-\Delta C_{\text {loss }}(k),
$$

where $\Delta C_{\text {gain }}(k)$ denotes the sum rate increment in the $i$ th cell by adding the $k$ th user to $S_{i}$, and $\Delta C_{\text {loss }}(k)$ denotes the sum rate decrement in adjacent cells by adding the $k$ th user to $S_{i}$ due to the increased interference. The BS can easily calculate $\Delta C_{\text {gain }}(k)$ as

$$
\Delta C_{\text {gain }}(k)=\sum_{k^{\prime} \in \mathbf{S}_{i} \cup\{k\}} r_{i}^{\left(k^{\prime}\right)}-\sum_{k^{\prime} \in \mathbf{S}_{i}} r_{i}^{\left(k^{\prime}\right)} .
$$

However, it is difficult to calculate $\Delta C_{\text {loss }}(k)$ in the distributed manner, since $\Delta C_{\text {loss }}(k)$ is dependent on the set of selected users in adjacent cells. Instead of directly calculating $\Delta C_{\text {loss }}(k)$, we propose to estimate $\Delta C_{\text {loss }}(k)$ based on $\beta_{i}^{(k)}$ which is fed back from the $k$ th MS in the $i$ th cell. Note that $\beta_{i}^{(k)}$ represents the amount of interference caused to adjacent cells by selecting the $k$ th user in the $i$ th cell. The main idea is to estimate $\Delta C_{\text {loss }}(k)$ by calculating the sum rate decrement in the ith cell to which the BS belongs, with additional interference with the power $\beta_{i}^{(k)}$. Then the estimated sum rate decrement $\Delta \tilde{C}_{\text {loss }}(k)$ in adjacent cell can be expressed as

$$
\Delta \tilde{C}_{\text {loss }}(k)=\sum_{k^{\prime} \in \mathbf{S}_{i} \cup\{k\}} r_{i}^{\left(k^{\prime}\right)}-\sum_{k^{\prime} \in \mathbf{S}_{i} \cup\{k\}} \tilde{r}_{i}^{\left(k^{\prime}\right)}\left(\beta_{i}^{(k)}\right),
$$

where $\tilde{r}_{i}^{\left(k^{\prime}\right)}\left(\beta_{i}^{(k)}\right)$ denotes the achievable rate of the $k^{\prime}$ th user in the $i$ th cell with additional interference of the power $\beta_{i}^{(k)}$, and it can be calculated as

$$
\tilde{r}_{i}^{\left(k^{\prime}\right)}\left(\beta_{i}^{(k)}\right)=\log \left(1+\overline{\operatorname{SINR}}_{i}^{\left(k^{\prime}\right)}\left(\beta_{i}^{(k)}\right)\right),
$$

where

$$
\begin{aligned}
\overline{\operatorname{SINR}}_{i}^{\left(k^{\prime}\right)}\left(\beta_{i}^{(k)}\right)= & \left(\sqrt{\rho_{i}^{\left(k^{\prime}\right)}} \mathbf{H}_{i, i}^{\left(k^{\prime}\right)} \mathbf{w}_{i}^{\left(k^{\prime}\right)}\right)^{H}\left(\mathbf{K}_{\mathrm{NI}}^{\left(i, k^{\prime}\right)}\left(\beta_{i}^{(k)}\right)\right)^{-1} \\
& \times\left(\sqrt{\rho_{i}^{\left(k^{\prime}\right)}} \mathbf{H}_{i, i}^{\left(k^{\prime}\right)} \mathbf{w}_{i}^{\left(k^{\prime}\right)}\right),
\end{aligned}
$$

$$
\begin{aligned}
\tilde{\mathbf{K}}_{\mathrm{NI}}^{\left(i, k^{\prime}\right)}\left(\beta_{i}^{(k)}\right)= & \left(1+\frac{N_{r}}{\beta_{i}^{(k)}}\right) \mathbf{I}_{N_{r}}+\sum_{k^{\prime} \in \mathbf{S}_{i}, k^{\prime} \neq k} \rho_{i}^{\left(k^{\prime}\right)}\left(\mathbf{H}_{i, i}^{\left(k^{\prime}\right)} \mathbf{w}_{i}^{\left(k^{\prime}\right)}\right) \\
& \times\left(\mathbf{H}_{i, i}^{\left(k^{\prime}\right)} \mathbf{w}_{i}^{\left(k^{\prime}\right)}\right)^{H}+\sum_{j=1, j \neq i}^{L} \sum_{k^{\prime} \in \mathbf{S}_{j}} \eta_{i, j}^{\left(k^{\prime}\right)}\left(\mathbf{H}_{i, j}^{\left(k^{\prime}\right)} \mathbf{w}_{j}^{\left(k^{\prime}\right)}\right) \\
& \times\left(\mathbf{H}_{i, j}^{\left(k^{\prime}\right)} \mathbf{w}_{j}^{\left(k^{\prime}\right)}\right)^{H} .
\end{aligned}
$$

From (15) and (16), the estimated $\Delta C(k)$ can be obtained as

$$
\begin{aligned}
\Delta \tilde{C}(k) & =\Delta C_{\text {gain }}(k)-\Delta \tilde{C}_{\text {loss }}(k) \\
& =\sum_{k^{\prime} \in \mathbf{S}_{i} \cup\{k\}} \tilde{r}_{i}^{\left(k^{\prime}\right)}\left(\beta_{i}^{(k)}\right)-\sum_{k^{\prime} \in \mathbf{S}_{i}} r_{i}^{\left(k^{\prime}\right)} .
\end{aligned}
$$

The proposed algorithm requires at most $K N_{r}$ computations of $\Delta \tilde{C}(k)$ per cell, since users are successively selected.

\section{Proportional fairness}

The proportional fairness (PF) scheduling effectively provides a trade-off between the average throughput and fairness among users [11]. The conventional PF scheduling was originally proposed for a single cell environment. In this case, each BS selects users as

$$
\begin{aligned}
\mathbf{S}_{\mathrm{CONV} i} & =\underset{\mathbf{S}_{i}}{\arg \max } \sum_{k=1}^{K} \log \left(\bar{R}_{i}^{(k)}\right) \text { for } i \\
& =1,2, \ldots, L,
\end{aligned}
$$

where $\bar{R}_{i}^{(k)}$ denotes the average throughput estimate of the $k$ th user in the $i$ th cell. We assume that $\bar{R}_{i}^{(k)}$ is calculated as

$$
\bar{R}_{i}^{(k)}(t)=\left\{\begin{array}{cl}
\left(1-\frac{1}{T_{c}}\right) \bar{R}_{i}^{(k)}(t-1)+\frac{1}{T_{c}} r_{i}^{(k)}(t), & \text { if served at } t \\
\left(1-\frac{1}{T_{c}}\right) \bar{R}_{i}^{(k)}(t-1), & \text { if not served at } t
\end{array}\right.
$$

where $T_{\mathrm{c}}$ is the time constant of the averaging window. The solution of (21), however, does not guarantee the system-wide PF due to the intercell interference.

We consider an optimal user selection criterion for the system-wide PF, which can be expressed as

$$
\left(\mathbf{S}_{\mathrm{opt} 1}, \mathbf{S}_{\mathrm{opt} 2}, \ldots, \mathbf{S}_{\mathrm{opt} L}\right)=\underset{\left(\mathbf{S}_{1}, \mathbf{S}_{2}, . . \mathbf{S}_{L}\right)}{\arg \max }\left(U_{1}\right),
$$

where $U_{1}$ is the system-wide PF utility function expressed as

$$
U_{1}=\sum_{i=1}^{L} \sum_{k=1}^{K} \log \left(\bar{R}_{i}^{(k)}\right)
$$

As in (13), the optimal solution of (23) needs centralized optimization among cells. Here, we also propose a suboptimal distributed algorithm. Instead of $U_{1}$ in (23), we use another utility function $U_{2}$ given as

$$
U_{2}=\prod_{i=1}^{L} \prod_{k \in \mathbf{S}_{i}}\left(1+\frac{1}{T_{c}-1} \frac{r_{i}^{(k)}}{\bar{R}_{i}^{(k)}}\right) .
$$


As provided in the Appendix, the optimization problem (23) remains the same even though $U_{1}$ is replaced with $U_{2}$. The use of $U_{2}$ enables the user selection algorithm to work in a distributed fashion with low computational complexity. Based on the newly defined utility function $U_{2}$, the proposed algorithm works as follows.

Step 1. Initialization: $\mathbf{S}_{i}=\{\}$.

Step 2. $k_{\text {new }}=\arg \max _{k} \Delta U_{2}(k)$.

Step 3. If $\Delta U_{2}\left(k_{\text {new }}\right)>0$, then $\mathbf{S}_{i}=\mathbf{S}_{i} \cup\left\{k_{\text {new }}\right\}$ and go

back to the Step 2, otherwise terminate the algorithm.

Note that the above algorithm is the same as the distributed algorithm developed in Section 4.1, except that $\Delta C(k)$ is replaced by $\Delta U_{2}(k)$, which denotes the amount of the change in $U_{2}$ when the $k$ th user is added to $S i$.

As in (14), $\Delta U_{2}(k)$ can be expressed as

$$
\Delta U_{2}(k)=\Delta U_{2 \text { gain }}(k)-\Delta U_{2 \text { loss }}(k),
$$

where $\Delta U_{\text {2gain }}(k)$ denotes the increment of $U_{2}$ in the $i$ th cell by adding the $k$ th user to $S_{i}$, which can be expressed as

$$
\begin{aligned}
\Delta U_{2 \text { gain }}(k)= & \prod_{k^{\prime} \in \mathbf{S}_{i} \cup\{k\}}\left(1+\frac{1}{T_{c}-1} \frac{r_{i}^{\left(k^{\prime}\right)}}{\bar{R}_{i}^{\left(k^{\prime}\right)}}\right) \\
& -\prod_{k^{\prime} \in \mathbf{S}_{i}}\left(1+\frac{1}{T_{c}-1} \frac{r_{i}^{\left(k^{\prime}\right)}}{\bar{R}_{i}^{\left(k^{\prime}\right)}}\right) .
\end{aligned}
$$

$\Delta U_{2 \text { loss }}(k)$ in (26) denotes the decrement of $U_{2}$ in adjacent cells by adding the $k$ th user to $S_{i}$ due to the increased interference. Like the approach used for the total sum rate maximization, we propose to estimate $\Delta U_{2 \text { loss }}(k)$ as

$$
\begin{aligned}
\Delta \tilde{U}_{\text {2loss }}(k)= & \prod_{k^{\prime} \in \mathbf{S}_{i} \cup\{k\}}\left(1+\frac{1}{T_{c}-1} \frac{r_{i}^{\left(k^{\prime}\right)}}{\bar{R}_{i}^{\left(k^{\prime}\right)}}\right) \\
& -\prod_{k^{\prime} \in \mathbf{S}_{i} \cup\{k\}}\left(1+\frac{1}{T_{c}-1} \frac{\tilde{r}_{j}^{\left(k^{\prime}\right)}\left(\beta_{i}^{(k)}\right)}{\bar{R}_{i}^{\left(k^{\prime}\right)}}\right) .
\end{aligned}
$$

Then, by using (27) and (28), the estimation of $\Delta U_{2}(k)$ can be found as

$$
\begin{aligned}
\Delta \tilde{U}_{2}(k)= & \prod_{k^{\prime} \in \mathbf{S}_{i} \cup\{k\}}\left(1+\frac{1}{T_{c}-1} \frac{\tilde{r}_{j}^{\left(k^{\prime}\right)}\left(\beta_{i}^{(k)}\right)}{\bar{R}_{i}^{\left(k^{\prime}\right)}}\right) \\
& -\prod_{k^{\prime} \in \mathbf{S}_{i}}\left(1+\frac{1}{T_{c}-1} \frac{r_{j}^{\left(k^{\prime}\right)}}{\bar{R}_{i}^{\left(k^{\prime}\right)}}\right) .
\end{aligned}
$$

This algorithm also requires at most $K N_{r}$ computations of $\Delta \tilde{U}_{2}(k)$ per cell.

\section{Simulation results}

In this section, we evaluate the performance of the transmit beamforming and user selection algorithms discussed in Sections 3 and 4 using computer simulations. We consider a wrap-around hexagonal model with seven cells as shown in Figure 2. There are $K$ users per cell who are assumed to be uniformly distributed over the cell. Each channel between the MS and BS is assumed to experience an independent long-term fading comprised of the path loss and log-normal shadow fading. Correspondingly, $\rho_{i}^{(k)}$ and $\eta_{i, j}^{(k)}$ in (1) can be expressed as

$$
\begin{aligned}
& \rho_{i}^{(k)}=10^{\frac{s_{i, i}^{(k)}}{10}}\left(d_{i, i}^{(k)}\right)^{-\alpha} P_{i}^{(k)}, \\
& \eta_{i, j}^{(k)}=10^{s_{i, j}^{(k)}}\left(d_{i, j}^{(k)}\right)^{-\alpha} P_{j}^{(k)},
\end{aligned}
$$

where $d_{i, j}^{(k)}$ is the distance between the BS in the $i$ th cell and the $k$ th MS in the $j$ th cell, $\alpha$ is the path loss exponent, and $s_{i, i}^{(k)}$ is a zero-mean Gaussian random variable that stands for the shadow fading. It is assumed that the long-term power control perfectly compensates for the long-term fading so that a given target SNR is satisfied at the BS. In the following simulation, the path loss exponent, $\log$ standard deviation of the shadow fading, and the target $\mathrm{SNR}$ are set to $3.7,8 \mathrm{~dB}$, and $10 \mathrm{~dB}$, respectively.

We first consider user selection for the sum rate maximization. Figures 3 and 4 depict the average achievable sum rate per cell versus the number of users for

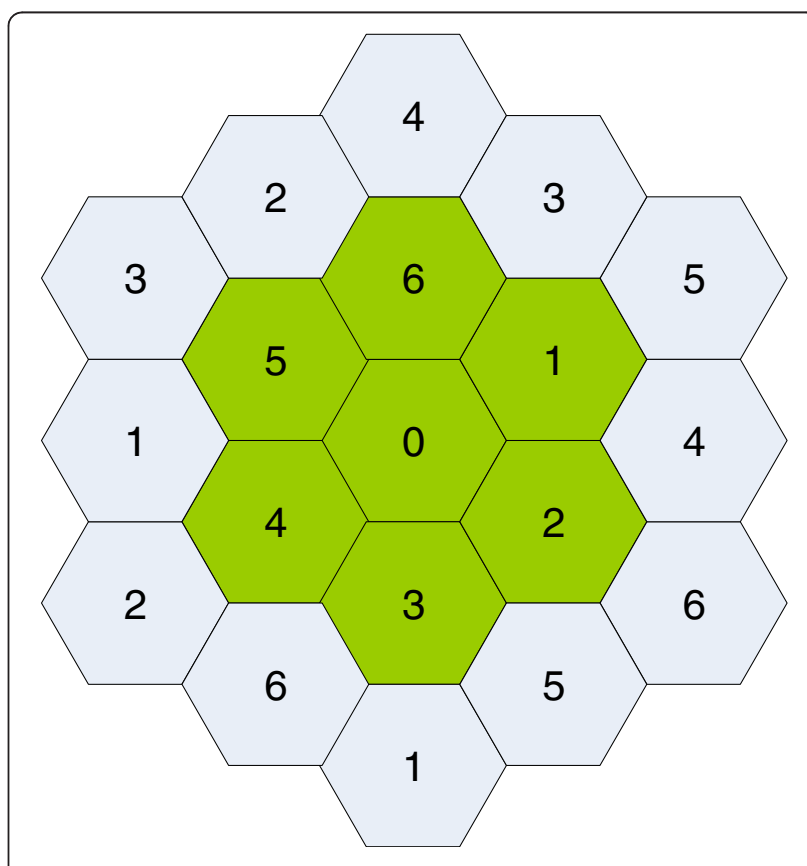

Figure 2 Wrap-around hexagonal model with seven cells. 


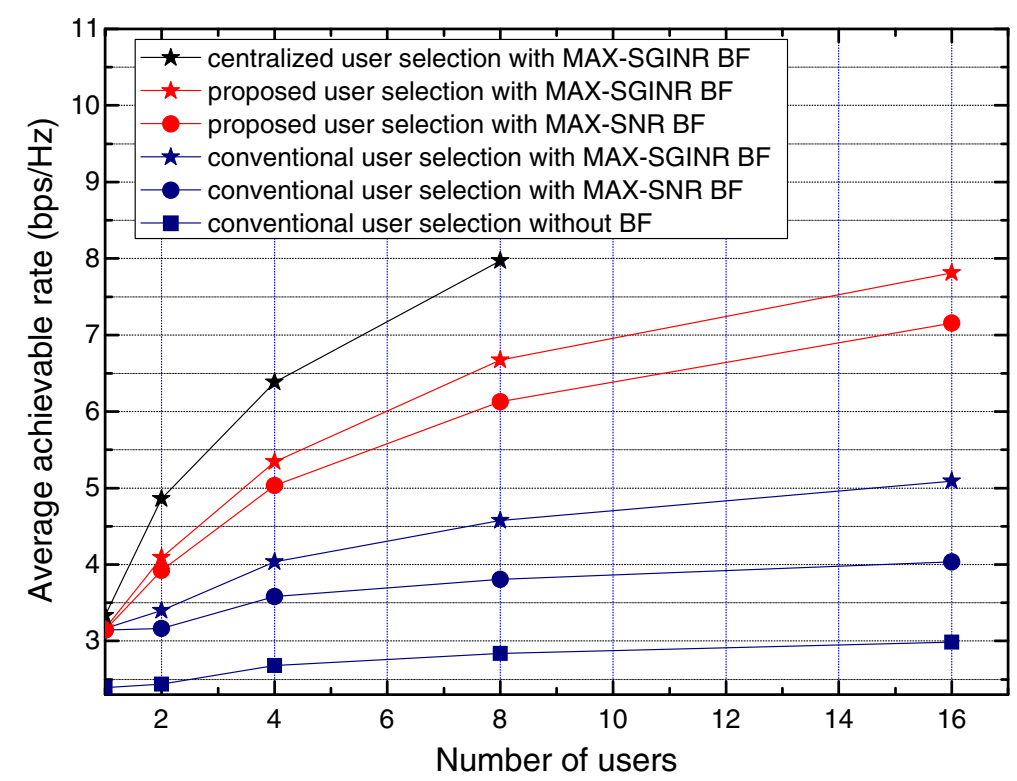

Figure 3 The average achievable sum rate per cell versus the number of users for $N_{t}=N_{r}=2$.

$N_{t}=N_{r}=2$ and $N_{t}=N_{r}=4$, respectively. The performance of the centralized user selection derived from an exhaustive search is plotted together as an upper bound. However, the results are provided only up to eight users due to very high computational complexity. It is shown that the MAX-SGINR beamforming outperforms the MAX-SNR beamforming, and the proposed user selection scheme outperforms the conventional one. It must be noted that the proposed user selection gain increases with the number of users, and that the gain is more distinguished than the beamforming gain. For the case of $K=16$ and $N_{t}=N_{r}=2$, for example, the proposed user selection scheme is shown to provide as much as $2.72 \mathrm{bps} / \mathrm{Hz}$ improvement over the conventional user selection scheme, when the MAX-SGINR beamforming is adopted. Under the same conditions, the gain of the

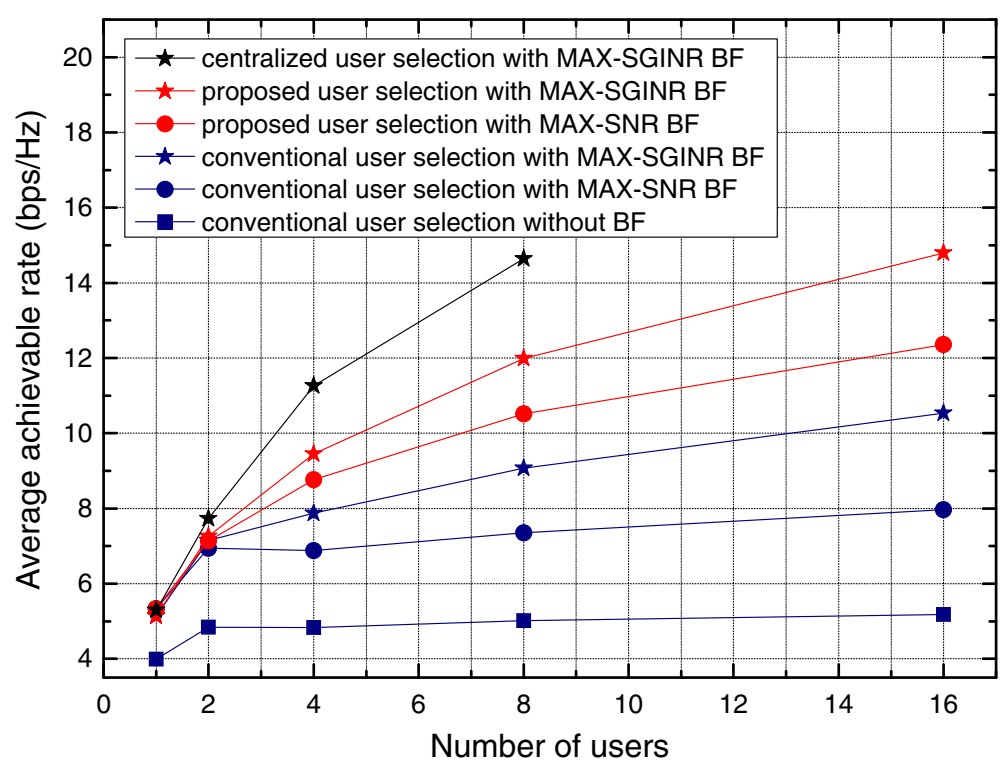

Figure 4 The average achievable sum rate per cell versus the number of users for $N_{t}=N_{r}=4$. 


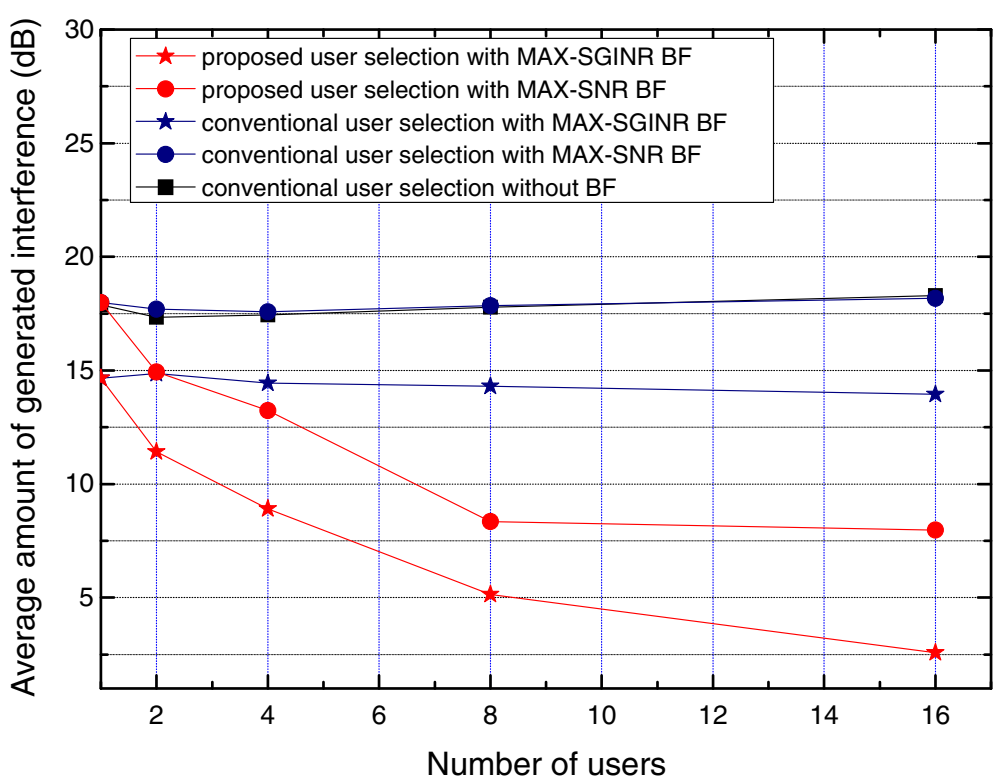

Figure 5 The average amount of generated interference per cell versus the number of users for $N_{t}=N_{r}=2$.

MAX-SGINR beamforming over the MAX-SNR beamforming is $0.65 \mathrm{bps} / \mathrm{Hz}$, when the proposed user selection scheme is applied. Figure 5 depicts the average amount of generated interference per cell for $N_{t}=N_{r}=2$. It is shown that the proposed user selection scheme considerably reduces the generated interference especially for a large number of users.

Now we consider the case of the PF utility. Figures 6 and 7 depict the system-wide PF utility $U_{1}$ and the average achievable sum rate per cell, respectively, versus time for $K=16, N_{t}=N_{r}=2$, and $T_{c}=200$ slots. As in the case of the sum rate maximization, the MAX-SGINR beamforming outperforms the MAX-SNR beamforming, and the proposed user selection scheme outperforms the conventional one. The results in Figure 6 also imply that the proposed scheme improves the fairness among users as compared to the conventional scheme. Correspondingly, the proposed user selection scheme

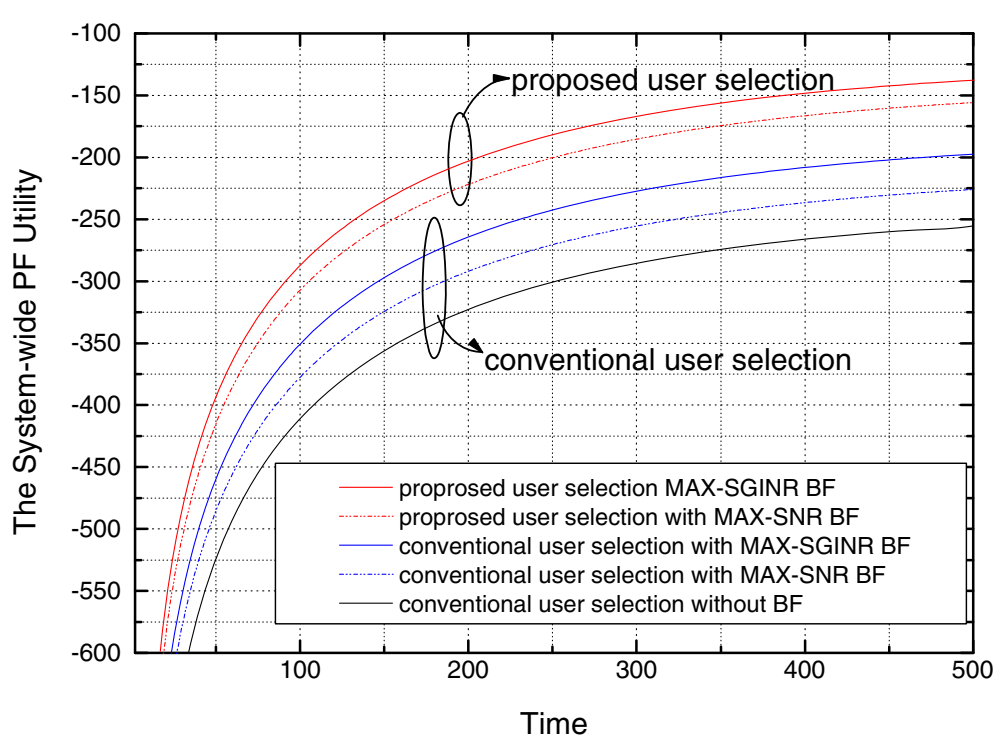

Figure 6 The system-wide PF utility versus time for $K=16$ and $N_{t}=N_{r}=2$. 


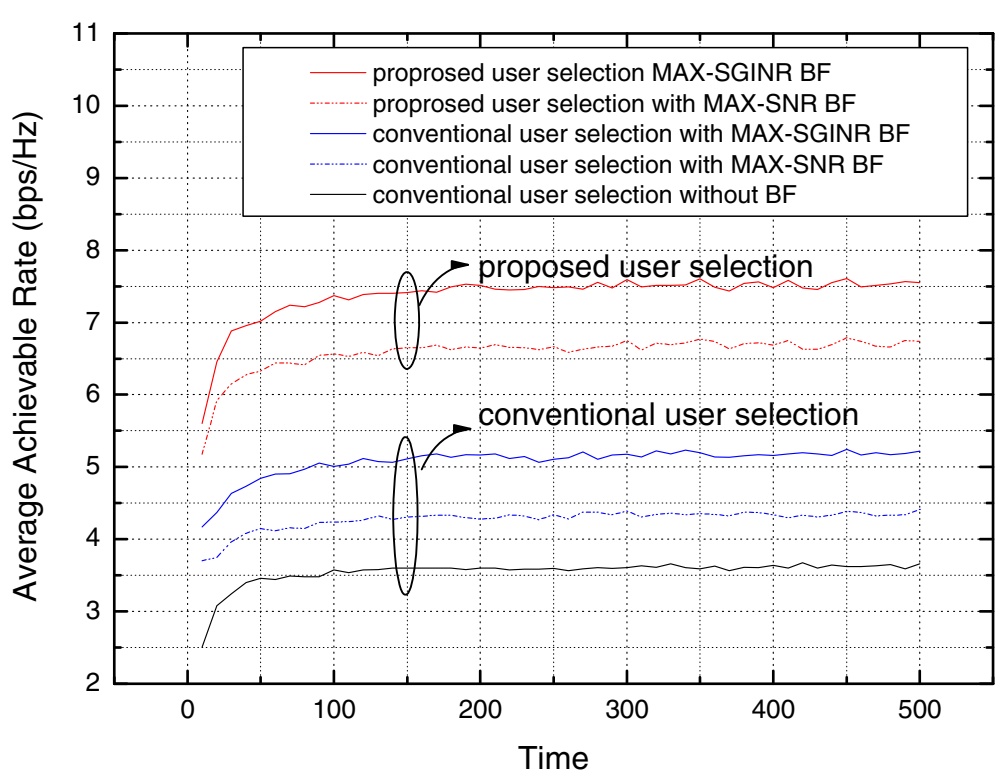

Figure 7 The average achievable sum rate per cell versus time for $K=16$ and $N_{t}=N_{r}=2$.

with the MAX-SGINR beamforming provides the best performance.

\section{Conclusion}

In this article, we have developed an interference-aware distributed user selection scheme for uplink MU-MIMO systems in a multicell environment. Multiple transmit antennas at each MS are utilized for transmit beamforming to reduce the interference caused to adjacent cells. Multiple receive antennas at each BS are utilized for receiving the signals from the selected users and suppressing intercell interference. We have derived system-wide optimal user selection criteria and proposed distributed user selection algorithms with low complexity. Simulation results have shown that the proposed user selection scheme provides significant performance improvement in a multicell environment.

\section{Appendix}

$$
\begin{aligned}
& \left(\mathbf{S}_{\mathrm{opt} 1}, \mathbf{S}_{\mathrm{opt} 2}, . . \mathbf{S}_{\mathrm{opt} L}\right)=\underset{\left(\mathbf{S}_{1}, \mathbf{S}_{2}, . . \mathbf{S}_{L}\right)}{\arg \max }\left(U_{1}\right) \\
& =\underset{\left(\mathbf{S}_{1}, \mathbf{S}_{2}, . . \mathbf{S}_{L}\right)}{\arg \max } \sum_{i=1}^{L} \sum_{k=1}^{K} \log \bar{R}_{i}^{(k)}(t) \\
& =\underset{\left(\mathbf{S}_{1}, \mathbf{S}_{2}, . . \mathbf{S}_{L}\right)}{\arg \max }\left(\sum_{i=1}^{L} \sum_{k \in \mathbf{S}_{i}} \log \bar{R}_{i}^{(k)}(t)+\sum_{i=1}^{L} \sum_{k \notin \mathbf{S}_{i}} \log \bar{R}_{i}^{(k)}(t)\right)
\end{aligned}
$$

$$
\begin{aligned}
= & \underset{\left(\mathbf{S}_{1}, \mathbf{S}_{2}, . . \mathbf{S}_{L}\right)}{\arg \max }\left(\sum_{i=1}^{L} \sum_{k \in \mathbf{S}_{i}} \log \left(\left(1-\frac{1}{T_{c}}\right) \bar{R}_{i}^{(k)}(t-1)+\frac{1}{T_{c}} r_{i}^{(k)}\right)+\sum_{i=1}^{L} \sum_{k \notin \mathbf{S}_{i}}\left(1-\frac{1}{T_{c}}\right) \bar{R}_{i}^{(k)}(t-1)\right) \\
= & \underset{\left(\mathbf{S}_{1}, \mathbf{S}_{2}, . . \mathbf{S}_{L}\right)}{\arg \max }\left(\begin{array}{l}
\left.\sum_{i=1}^{L} \sum_{k \in \mathbf{S}_{i}} \log \left(1-\frac{1}{T_{c}}\right) \bar{R}_{i}^{(k)}(t-1)+\sum_{i=1}^{L} \sum_{k \in \mathbf{S}_{i}} \log \left(1+\frac{1}{T_{c}-1} \frac{r_{i}^{(k)}}{\bar{R}_{i}^{(k)}(t-1)}\right)\right) \\
+\sum_{i=1}^{L} \sum_{k \notin \mathbf{S}_{i}}\left(1-\frac{1}{T_{c}}\right) \bar{R}_{i}^{(k)}(t-1)
\end{array}\right) \\
= & \underset{\left(\mathbf{S}_{1}, \mathbf{S}_{2}, . . \mathbf{S}_{L}\right)}{\arg \max }\left(\sum_{i=1}^{L} \sum_{k=1}^{K} \log \left(1-\frac{1}{T_{c}}\right) \bar{R}_{i}^{(k)}(t-1)+\sum_{i=1}^{L} \sum_{k \in \mathbf{S}_{i}} \log \left(1+\frac{1}{T_{c}-1} \frac{r_{i}^{(k)}}{\bar{R}_{i}^{(k)}(t-1)}\right)\right)
\end{aligned}
$$




$$
\begin{aligned}
& =\underset{\left(\mathbf{S}_{1}, \mathbf{S}_{2}, . . \mathbf{S}_{L}\right)}{\arg \max } \sum_{i=1}^{L} \sum_{k \in \mathbf{S}_{i}} \log \left(1+\frac{1}{T_{c}-1} \frac{r_{i}^{(k)}}{\bar{R}_{i}^{(k)}(t-1)}\right) \\
& =\underset{\left(\mathbf{S}_{1}, \mathbf{S}_{2}, . . \mathbf{S}_{L}\right)}{\arg \max } \prod_{i=1}^{L} \prod_{k \in \mathbf{S}_{i}}\left(1+\frac{1}{T_{c}-1} \frac{r_{i}^{(k)}}{\bar{R}_{i}^{(k)}(t-1)}\right) \\
& =\underset{\left(\mathbf{S}_{1}, \mathbf{S}_{2}, . . \mathbf{S}_{L}\right)}{\arg \max }\left(U_{2}\right) .
\end{aligned}
$$

\section{Competing interests}

The authors declare that they have no competing interests.

\section{Acknowledgment}

This work was supported in part by the National Research Foundation of Korea (NRF) grant funded by the Korea government (MEST) (No. 20090085604), and in part by the KCC (Korea Communications Commission), Korea, under the R\&D program supervised by the KCA (Korea Communications Agency) (KCA-2011-08911-04003).

\section{Author details}

'Modem System Lab, Samsung Electronics, Suwon 443-742, South Korea. ${ }^{2}$ School of Electronic Engineering, Soongsil University, Seoul 156-743, South Korea.

${ }^{3}$ School of Electrical Engineering \& Computer Science, Seoul National University, Seoul 151-742, South Korea.

Received: 4 February 2012 Accepted: 12 May 2012

Published: 21 June 2012

\section{References}

1. K.N. Lau, Analytical framework for multiuser uplink MIMO space-time scheduling design with convex utility functions. IEEE Trans. Wirel. Commun. 3(9), 1832-1843 (2004)

2. Y. Hara, L. Brunel, K. Oshima, Uplink spatial scheduling with adaptive transmit beamforming in multiuser MIMO systems, in Proceeding of IEEE International Symposium on Personal, Indoor and Mobile Radio Communications, Helsinki, Finland, September 2006.. doi:10.1109/ PIMRC.2006.254006

3. S. Serbetli, A. Yener, Beamforming and scheduling strategies for time slotted multiuser MIMO systems, in Proceeding of Asilomar Conference on Signals, Systems, and Computers, Pacific Grove, CA USA, 1st edn., 2004, pp. 1227-1231

4. S. Catreux, P.F. Driessen, L.J. Greenstein, Simulation results for an interference-limited multiple-input multiple-output cellular system. IEEE Commun. Lett. 4(11), 334-336 (2000)

5. R.S. Blum, MIMO capacity with interference. IEEE J. Sel. Areas Commun. 21 (6), 793-801 (2003)

6. S. Ye, R.S. Blum, Optimized signaling for MIMO interference systems with feedback. IEEE Trans. Signal Process. 51(11), 2939-2848 (2003)

7. B.O. Lee, H.W. Je, O.S. Shin, K.B. Lee, A novel uplink MIMO transmission scheme in a multicell environment. IEEE Trans. Wirel. Commun. 8(10), 49814987 (2009)

8. S.A. Ramprashad, H.C. Papadopoulos, A. Benjebbour, Y. Kishiyama, N. Jindal, G. Caire, Cooperative cellular networks using multi-user MIMO: tradeoffs, overheads, and interference control across architectures. IEEE Commun. Mag. 49(5), 70-77 (2011)

9. M. Kobayashi, M. Debbah, J. Belfiore, Outage efficient strategies for network MIMO with partial CSIT, in Proceeding of IEEE International Symposium on Information Theory (, Seoul, Korea, 2009), pp. 249-253. doi:10.1109/ ISIT.2009.5206071

10. J. Hoydis, M. Kobayashi, M. Debbah, Optimal channel training in uplink network MIMO systems. IEEE Trans. Signal Process. 59(6), 2824-2833 (2011)
11. A. Jalali, R. Padovani, R. Pankaj, Data throughput of CDMA-HDR a high efficiency-high data rate personal communication wireless system, in Proceeding of IEEE Vehicular Technology Conference-Spring (, Tokyo, Japan, 2000), pp. 1854-1858. vol. 3

doi:10.1186/1687-1499-2012-202

Cite this article as: Lee et al.: Distributed user selection scheme for uplink multiuser MIMO systems in a multicell environment. EURASIP Journal on Wireless Communications and Networking 2012 2012:202.

\section{Submit your manuscript to a SpringerOpen ${ }^{\circ}$ journal and benefit from:}

- Convenient online submission

- Rigorous peer review

- Immediate publication on acceptance

- Open access: articles freely available online

- High visibility within the field

- Retaining the copyright to your article

Submit your next manuscript at $\gg$ springeropen.com 